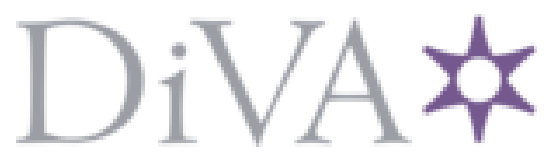

http://www.diva-portal.org

This is the published version of a paper published in Applied Physics Letters.

Citation for the original published paper (version of record):

Yao, M., Fan, X., Zhang, W., Bao, Y., Liu, R. et al. (2017)

Uniaxial-stress-driven transformation in cold compressed glassy carbon

Applied Physics Letters, 111(10): 101901

https://doi.org/10.1063/1.4996278

Access to the published version may require subscription.

N.B. When citing this work, cite the original published paper.

Applied Physical Letters: Publishers version/PDF may be used on author's personal website, arXiv, institutional website, institutional repository, funders designated repository or private forums on social academic network after 12 months embargo.

Permanent link to this version:

http://urn.kb.se/resolve?urn=urn:nbn:se:umu:diva-140045 


\section{Uniaxial-stress-driven transformation in cold compressed glassy carbon}

Cite as: Appl. Phys. Lett. 111, 101901 (2017); https://doi.org/10.1063/1.4996278

Submitted: 15 July 2017 . Accepted: 25 August 2017 . Published Online: 06 September 2017

Mingguang Yao, Xianhong Fan, Weiwei Zhang (D), Yongjun Bao (D), Ran Liu, Bertil Sundqvist (iD), and Bingbing Liu
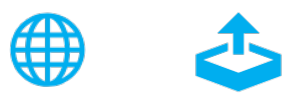

\section{ARTICLES YOU MAY BE INTERESTED IN}

Variable range hopping electric and thermoelectric transport in anisotropic black phosphorus Applied Physics Letters 111, 102101 (2017); https://doi.org/10.1063/1.4985333

Impact of defect distribution on $\mathrm{IrO}_{\mathrm{X}} / \mathrm{ZnO}$ interface doping and Schottky barriers Applied Physics Letters 111, 101604 (2017); https://doi.org/10.1063/1.4989539

Resistive spectroscopy coupled with non-contacting oscillator for detecting discontinuouscontinuous transition of metallic films

Applied Physics Letters 111, 101902 (2017); https://doi.org/10.1063/1.4995469 


\title{
Uniaxial-stress-driven transformation in cold compressed glassy carbon
}

\author{
Mingguang Yao, ${ }^{1,2, a)}$ Xianhong Fan, ${ }^{2}$ Weiwei Zhang, ${ }^{1}$ Yongjun Bao, ${ }^{1}$ Ran Liu, ${ }^{1}$ \\ Bertil Sundqvist, ${ }^{1,3}$ and Bingbing Liu ${ }^{1, a)}$ \\ ${ }^{1}$ State Key Laboratory of Superhard Materials, Jilin University, Changchun 130012, China \\ ${ }^{2}$ College of Physics, Jilin University, Changchun 130012, China \\ ${ }^{3}$ Department of Physics, Umea University, S-90187 Umea, Sweden
}

(Received 15 July 2017; accepted 25 August 2017; published online 6 September 2017)

\begin{abstract}
We show that transformation of glassy carbon (GC) into a translucent superhard carbon phase by cold-compression is obtained in the presence of a uniaxial stress field. This transition accompanies with $\mathrm{sp}^{2}$ to $\mathrm{sp}^{3}$ bonding change in $\mathrm{GC}$, and it is found that the uniaxial stress strongly favors this bonding transition. The transformation of GC causes photoluminescence and significantly increases light transmissivity. Upon decompression, the high pressure phase can be maintained under large uniaxial stress at a chamber pressure even down to $\sim 10 \mathrm{GPa}$. We discuss possible mechanisms of these transitions by a distinct bonding process that occurs in noncrystalline carbon. Published by AIP Publishing. [http://dx.doi.org/10.1063/1.4996278]
\end{abstract}

Discovering phase transitions in materials and understanding their mechanisms are very important both from a fundamental perspective and for applications. This is particularly interesting in the investigations of the transformations of carbon materials under extreme conditions such as high pressure because various carbon allotropes with desired properties may be formed which cannot be obtained at ambient conditions thanks to the versatile bonding ability of carbon. The effect of structural order and topology on the evolution between $\mathrm{sp}^{2}$ and $\mathrm{sp}^{3}$ bonded carbon structures is a major topic. In fact, all forms of carbon appear to evolve towards the diamond structure ${ }^{1-4}$ under sufficiently high pressure and high temperature (HPHT). The well explored phase diagram of carbon at HPHT is exploited in the commercial production of diamond. Other $\mathrm{sp}^{3}$ structures, whose nature strongly depends on the topology and degree of order in the carbon precursor, can be stabilized at lower temperatures. ${ }^{5-9}$ For example, graphite transforms into a transparent phase, whose structure is still debated, at above $15 \mathrm{GPa}$ and room temperature. ${ }^{10-12}$ We recently show that carbon nanotubes filled with fullerene $\mathrm{C}_{70}$ molecules transform into a quenchable, full $\mathrm{sp}^{3}$ carbon phase with a monoclinic structure at above $60 \mathrm{GPa} .{ }^{13}$

Compared to the graphitic materials, noncrystalline carbons have recently attracted enormous attention due to their advantages under certain circumstances. Glassy carbon (GC), for example, a highly disordered form of carbon, has been widely used in industry. Different models have been proposed to describe the structure of GC, ${ }^{14-16}$ and the most broadly accepted representation is that it is built up from discrete fragments of curved $\mathrm{sp}^{2}$ carbon hexagonal sheets in which pentagons and heptagons are found to be intricately distributed. ${ }^{17}$ The conditions necessary for the evolution of GC towards a carbon $\mathrm{sp}^{3}$ hybridized system could be of particular interest when trying to create desired materials and understand the underlying transition mechanism for

\footnotetext{
${ }^{\text {a) }}$ Authors to whom correspondence should be addressed: yaomg@jlu.edu.cn and liubb@jlu.edu.cn
}

amorphous carbon, which is an important topic in materials science which is still far from fully understood. ${ }^{18,19}$

Glassy carbon transforms into nanocrystalline cubic diamond under HPHT, ${ }^{3}$ while it transforms into a class of mixed $\mathrm{sp}^{2}-\mathrm{sp}^{3}$ compressed GC materials with interesting properties at high pressure and moderate temperature,${ }^{20}$ but its transformations under cold compression are more complicated and still debated. The main controversies are as follows: whether there is a pressure-induced bonding change from $\mathrm{sp}^{2}$ to $\mathrm{sp}^{3}$ and whether a superhard noncrystalline phase forms in GC (or not) under pressure. ${ }^{21-23}$ Moreover, it is still a challenge to simulate the transitions of $\mathrm{GC}$ under pressure in theory because of the lack of long-range translational symmetry in such noncrystalline solids. Therefore, to clarify the transition of $\mathrm{GC}$ under pressure and understand the corresponding mechanism is urgent. ${ }^{24}$ Here, we report the results of a specially designed experiment showing that GC transforms reversibly into a $\mathrm{sp}^{3}$-bonded translucent phase. The experiment provides direct evidence that the transition is strongly facilitated when a strong uniaxial stress occurs in the compression conditions. The present results clarify the reasons for the discrepancies between previous works ${ }^{21-24}$ and are also important for the synthesis of noncrystalline superhard carbons with potential applications.

High-pressure Raman spectra of glassy carbon were recorded at room temperature using a spectrometer (Renishaw InVia) equipped with $632.8 \mathrm{~nm}$ and $830 \mathrm{~nm}$ exciting lasers. Diamonds with $300 \mu \mathrm{m}$ culets were used as anvils. Stainless steel gaskets, $\sim 50 \mu \mathrm{m}$ in thickness with holes $120 \mu \mathrm{m}$ in diameter, were used as high-pressure chambers for loading the samples. More details on the experimental setup can be found elsewhere. ${ }^{24}$ In the high pressure experiments, two or three GC spheres with different diameters (from several micrometers to $40 \mu \mathrm{m}$ ) and a ruby chip for pressure calibration, together with a pressure transmitting medium (PTM), were loaded into the sample chamber. In the experiments, either $\mathrm{KBr}$ or liquid $\mathrm{N}_{2}$ was used as PTM, which gave similar results, indicating that the PTM used is not the dominant factor responsible for the observed transformations in GC 

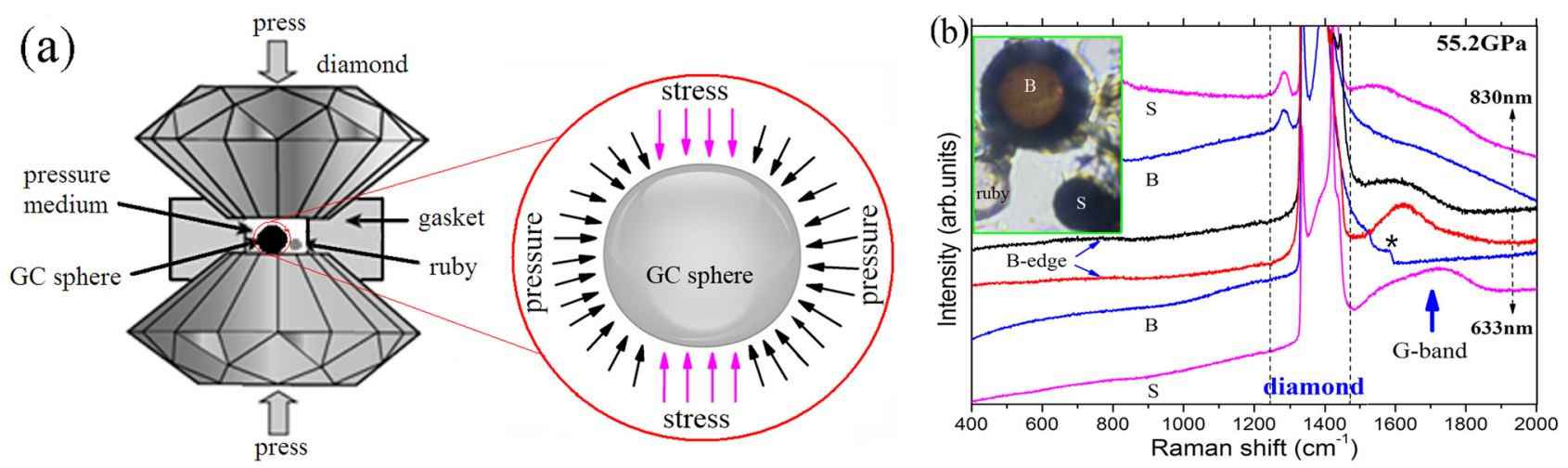

FIG. 1. (a) Sketch showing the sample loaded into the diamond anvil cell (DAC, left) and the combination of uniaxial stress and chamber pressure exerted on the sphere once it was compressed directly by two anvils (right). (b) Raman spectra from the translucent centres of the big ("B") and the opaque small ("S") spheres and from the opaque edge of the big sphere's ("B-edge"), at $55.2 \mathrm{GPa}$. Top two spectra were recorded using an excitation wavelength of $830 \mathrm{~nm}$, with bottom four spectra using $632.8 \mathrm{~nm}$. Note that the peak at $1282 \mathrm{~cm}^{-1}$ in the top two spectra is not from glassy carbon. The asterisk near $1580 \mathrm{~cm}^{-1}$ points to the position of the Raman line for the diamond-GC sphere contact point, which is used to calculate the uniaxial stress applied on GC. The inset shows an optical image of the spheres in DAC taken under back illumination. Liquid nitrogen was used as PTM.

spheres. X-ray diffraction (XRD) measurements were performed at the BL15U1 beamline at the Shanghai Synchrotron Radiation Facility $(\lambda=0.6199 \AA)$ at room temperature. One-dimensional diffraction patterns were obtained by integrating the two dimensional image with FIT2D software.

As shown in Fig. 1(a), when the large sphere is compressed directly between the two anvils under pressure, stress can be applied to the large GC sphere. This uniaxial stress $\left(\mathrm{P}_{\text {ind }}\right)$ is higher than the quasi-hydrostatic chamber pressure, and the pressure difference increases with increasing pressure as the two anvils approach each other and as the GC sphere itself hardens under deformation. Note that only when the large sphere was pre-compressed to become hard before the bridging and would not break with further compressing, the stress may happen to the sphere in our designed experiments. We found that the uniaxial stress strongly affects the transformation of GC spheres, and at some critical pressure, the center of GC spheres started to turn translucent. These critical pressures differed from experiment to experiment, depending on the magnitude of the stress. Figure 1(b) shows the results obtained at 55.2 GPa from one experiment. In this experiment, the center of the large sphere became translucent above $30 \mathrm{GPa}$, while the small sphere remains opaque during the compression. The pressure distribution above the translucent center of the sphere is homogeneous and is $\sim 113 \mathrm{GPa}$, as determined from the diamond Raman line because of the direct GC-diamond contact. ${ }^{25}$ The pressure above the opaque area near the sphere's periphery and above the small sphere measured by the same method is $\sim 55 \mathrm{GPa}$, which is almost the same as the chamber pressure $(\mathrm{Pr})$ calibrated by ruby. The large differential stress $(\Delta \mathrm{P})$ created in the large sphere by the uniaxial force from the two anvils thus caused a significant difference in the transformations of GC between the center and the edge of the large sphere and between the large and the small spheres.

Furthermore, we can show by Raman spectroscopy that there are differences in the bonding characteristics between the translucent and the opaque areas of the large spheres. As we can see from the Raman spectra shown in Fig. 1(b), the G-band of the translucent phase weakens significantly at such compression conditions and becomes hardly visible. As we know, the G-band of carbon materials is associated with the $\mathrm{C}-\mathrm{C}$ stretching mode in graphene layers and is a characteristic vibration for $\mathrm{sp}^{2}$ bonded $\mathrm{C} .{ }^{26}$ The disappearance of this band might indicate that $\mathrm{sp}^{2}$ carbon structures in the glassy carbon were almost transformed into $\mathrm{sp}^{3}$ bonded structures. Similar spectroscopic changes have also been observed in the experiment of compressing graphite, in which the G-band broadened significantly when the $\mathrm{sp}^{2}-\mathrm{sp}^{3}$ bonding change started and almost disappeared with increasing $\mathrm{sp}^{2}-\mathrm{sp}^{3}$ conversion. ${ }^{12}$ In contrast, the small sphere shows double bands in the G-band range, which preserves spectroscopic features of glassy carbon. It is interesting to note that the Raman spectra from the opaque part of the large sphere show one broad G-band, located at $1650 \mathrm{~cm}^{-1}$, which are different from glassy carbon but similar to those of graphite/ graphitic carbon materials under high pressure, indicating that the microstructures in the GC sphere were changed and graphitic phase might form. This may be due to the shifting

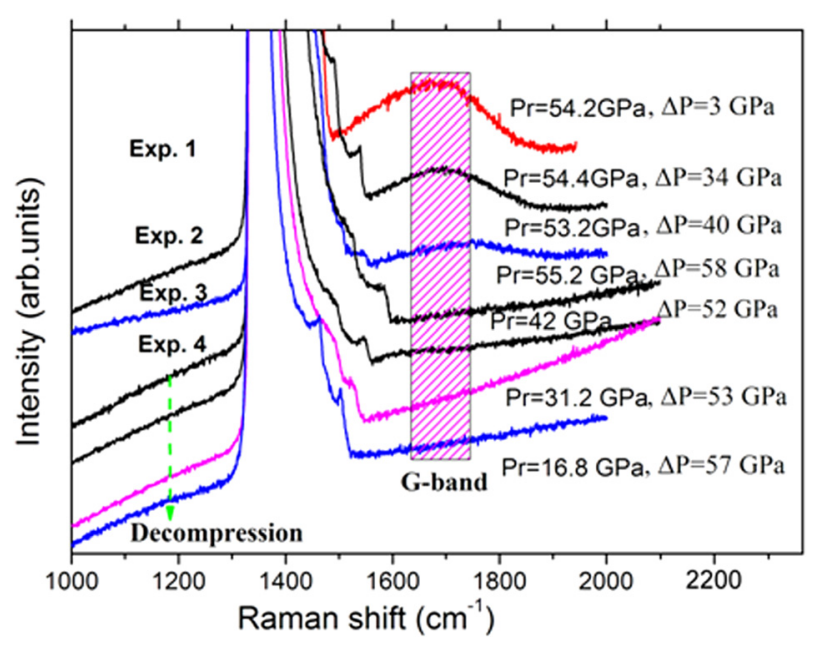

FIG. 2. Raman spectra of GC spheres under different pressure conditions in four separate experiments. The bottom four spectra are all from experiment 4 and were recorded at different pressures during decompression. Pr means the chamber pressure calibrated by ruby, while $\Delta \mathrm{P}$ represents the difference between $P_{r}$ and $P_{\text {ind }}$ (pressure at the GC-diamond contact area), calculated as $\Delta \mathrm{P}=\mathrm{P}_{\text {ind }}-\mathrm{Pr}$. 


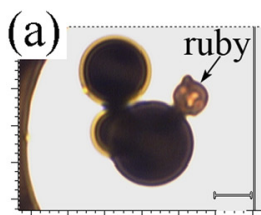

1. $3 \mathrm{GPa}$

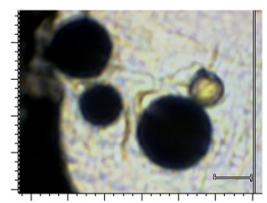

28. $9 \mathrm{GPa}$

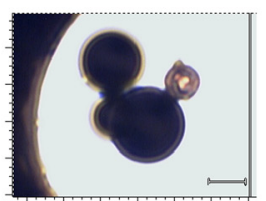

6. $1 \mathrm{GPa}$

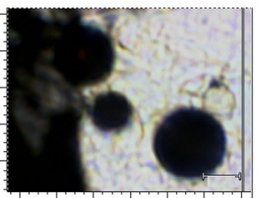

47. $1 \mathrm{GPa}$

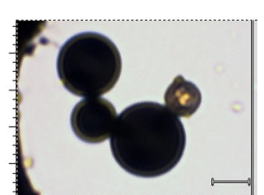

12. $2 \mathrm{GPa}$

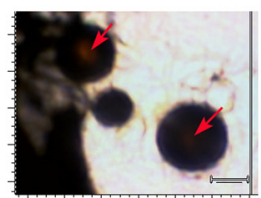

$53.4 \mathrm{GPa}$

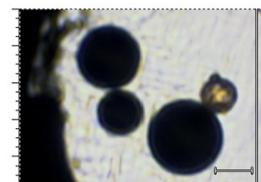

18. $0 \mathrm{GPa}$

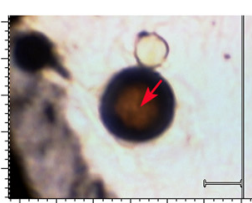

$\mathrm{d}-42.7 \mathrm{GPa}$

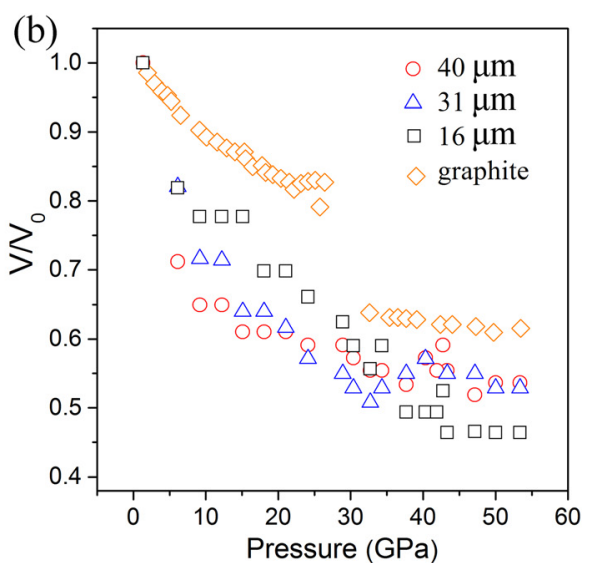

FIG. 3. (a) Optical images of three GC spheres at selected pressures in DAC. The last image corresponds to the sample decompressed to 42.7 GPa. The scale bar is $17 \mu \mathrm{m}$. All the photos were taken under back illumination. (b) Compression curves obtained by optical imaging in one experiment. We measured the diameter of the GC spheres and then estimated their volumes by the equation $\mathrm{V}=\frac{4}{3} \pi \mathrm{r}^{3}$, assuming a spherical shape of the spheres. The compression curve of graphite $^{12}$ is added for comparison.

of the graphene layers and responsible for the formation of the transparent phase in GC (see discussion in the last paragraph).

By a series of comparison experiments, we further find that the strength of the uniaxial stress strongly affects the G-band intensities of GC spheres under pressure. Figure 2 shows the Raman spectra collected from several experiments, in which the differences $\Delta \mathrm{P}$ between the uniaxial stress and the chamber pressure exerted on the GC had different magnitudes. Although the uniaxial stress depends on the sphere size, the chamber pressure, etc., it is clear that a larger $\Delta \mathrm{P}$ applied on the GC spheres always leads to a lower G-band intensity (Fig. S1 in the supplementary material), indicating a higher fraction of $\mathrm{sp}^{2}$ to $\mathrm{sp}^{3}$ carbon conversion in the compressed spheres. Remarkably, the large uniaxial stress created on the GC spheres and the translucent phase formed can be maintained upon decompression down to at least $17 \mathrm{GPa}$ (experiment 4). In addition, as described in previous work, ${ }^{22,24}$ the yield strength of the superhard GC spheres can be estimated using the calculated $\Delta \mathrm{P}$. Here, the very large stress difference $\left(\Delta \mathrm{P}_{\max } \sim 55 \mathrm{GPa}\right.$ in Fig. 2$)$ sustained by the GC sphere shows that its yield strength is comparable to that of diamond at ambient conditions.

As the densities of carbon materials are critical for their mechanical properties, we thus roughly estimated the volumetric compressions of the compressed GC spheres. Figure 3 shows optical images of GC spheres under pressure from one experiment. From these images, the volumetric compressions of the larger spheres and the small sphere are roughly calculated to be about $51 \%$ and $47 \%$ at $53.4 \mathrm{GPa}$, respectively, by simply analyzing the digital images and assuming an initial spherical shape [Fig. 3(b)]. For comparison, the volume per atom in compressed graphite at $\sim 55 \mathrm{GPa}$ decreases to $\sim 59 \%$ of the original value. ${ }^{12}$ The higher compressibility in glassy carbon should be related to the existence of large amounts of porosity in the material. Furthermore, the deformation of the large spheres due to the direct compression of diamond anvil is significant, while the small one almost preserves the spherical shape and remains opaque during the compression. Thus, the volumetric compression obtained in this way for the large one was underestimated. The initial bulk density of the type I GC used here is $\sim 1.54 \mathrm{~g} \mathrm{~cm}^{-3}$ and increases to $\sim 3.3 \mathrm{~g} / \mathrm{cm}^{3}$ for the small sphere at $53.4 \mathrm{GPa}$. The density of the compressed GC is comparable to that of diamond $\left(3.48 \mathrm{~g} / \mathrm{cm}^{3}\right)$. Our X-ray diffraction measurements on the sample confirm that the transformed GC with high density remains noncrystalline (Fig. S2 in the supplementary material).

We further characterize the optical properties of the translucent GC spheres by optical transmission and photoluminescence (PL) measurements. Figure 4(a) shows the transmission spectra of the large and small spheres at $44.8 \mathrm{GPa}$ after the transition and then upon decompression. It can be seen that the transmissivity of the incident light increases strongly when the GC spheres transform into translucent phases. The absorption band is broad with the center located at $650 \mathrm{~nm}$, and the broadening is consistent with the noncrystallinity of GC. The PL spectra of the two spheres are shown in Fig. 4(b). The PL peak position of both spheres is $\sim 660 \mathrm{~nm}$,
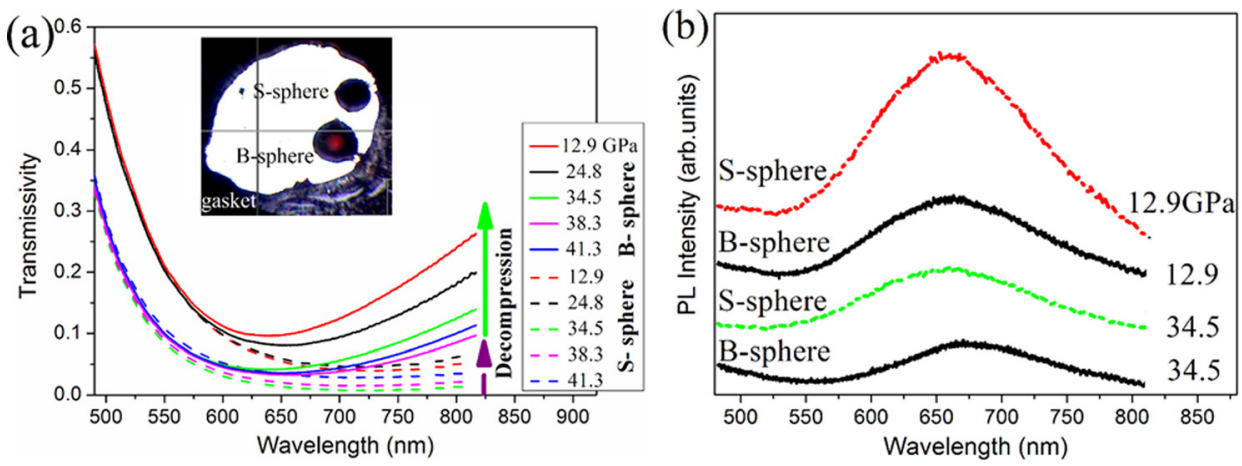

FIG. 4. (a) Optical transmission spectra of one big (B) and one small (S) GC sphere at different pressures. Spectra were taken during the decompression process at the pressures indicated. The inset shows an optical picture of the GC spheres in the gasket at $38.3 \mathrm{GPa}$. (b) Photoluminescence (PL) spectra of the GC spheres at selected pressures. 
which is close to that of the absorption peak energy. Moreover, the similar peak positions in PL and transmission spectra of both spheres suggest that the origin of the fluorescence and absorption in the two spheres should be the same, possibly due to the presence of conjugated $\pi$-systems (the unconverted $\mathrm{sp}^{2}$ carbons) distributed in the $\mathrm{sp}^{3}$ carbon matrix ${ }^{27}$ of the GC spheres under pressure. Under the same excitation conditions, the PL intensity of the small sphere is obviously higher than that of the big sphere, agreeing well with the fact that more $\mathrm{sp}^{2}$ carbons were preserved in the small spheres under pressure. Surprisingly, the translucent GC phase can be preserved upon decompression down to $\sim 10 \mathrm{GPa}$ (chamber pressure). The optical transmissivity of the sample increases with a slight downshift of the absorption peak energy upon decompression.

Let us now discuss the transition process of glassy carbon into the $\mathrm{sp}^{3}$ phase in our experiment. Upon compression, when uniaxial stress is absent in the experiment of compressing small GC spheres with diameters less than $10 \mu \mathrm{m}$, we found that no translucent phase was observed and the Raman spectra of the spheres still exhibited a broad and asymmetric band near $1800 \mathrm{~cm}^{-1}$ (similar to the spectra of opaque GC areas shown in Fig. 1) even up to $107 \mathrm{GPa}$ (chamber pressure). In this case, GC spheres are almost isotropically compressed and compacted, probably accompanied by minor sliding and shifting of the contained graphene fragments. From the spatial position point of view, only those $\mathrm{sp}^{2} \mathrm{C}$ atoms which are in appropriate positions, for example, short-ranged ordered carbon sheets distributed in $\mathrm{GC}$, could get converted into $\mathrm{sp}^{3}$ structures. This process is not sufficient to drive a complete $\mathrm{sp}^{3}$ carbon conversion in GC. Moreover, the $\mathrm{sp}^{3}$ structures formed in GC should be harder than the "unbounded" $\mathrm{sp}^{2}$ structures. Thus, a proper pre-compression could increase the stability of the GC spheres when submitted to large differential stress and favor the later formation of the $\mathrm{sp}^{3}$ phase. On the other hand, once the uniaxial stress increases on the GC spheres, according to previous literatures, ${ }^{28-30}$ a large pressure difference could generate a significant shear stress/strain. The shear zones should be concentrated in the weaker regions (the unbonded disordered areas), driving atomic displacements. This promotes the $\mathrm{sp}^{2}-\mathrm{sp}^{3}$ bonding change in GC and the formation of the $\mathrm{sp}^{3}$ superhard phase. It can thus be deduced that the $\mathrm{sp}^{3}$ bonding between $\mathrm{C}$ atoms in $\mathrm{GC}$ takes place in local areas at the primary compression stage and then spreads to the whole bulk under large uniaxial stress.

In summary, we have demonstrated that the transformation of glassy carbon into a translucent superhard phase is driven by a uniaxial stress field under cold compression. The $\mathrm{sp}^{2}$ to $\mathrm{sp}^{3}$ bond conversion in GC is very significant under high stress, causing PL phenomena and a significant increase in light transmissivity. It is important to note that the translucent phase can be preserved even down to around $10 \mathrm{GPa}$ upon decompression by maintaining a large uniaxial stress. The discovery of such an unexpected uniaxial-stress effect on the transformation in glassy carbon will stimulate further studies to search for analogous transitions and understand the corresponding mechanisms in other noncrystalline solids under compressions.

See supplementary materials for the analysis on X-ray diffraction data and the curve for the intensities of the "G-band" as a function of pressure difference for Fig. 2.

This work was supported financially by the National Natural Science Foundation of China (Nos. 11474121 and 51320105007) and the Fok Ying-Tong Education Foundation of China. The authors would like to thank Professor Alfonso San-Miguel from Lyon 1 University for fruitful discussions.

${ }^{1}$ T. Irifune, A. Kurio, S. Sakamoto, T. Inoue, and H. Sumiya, Nature 421, 599 (2003).

${ }^{2}$ Q. Huang, D. Yu, B. Xu, W. Hu, Y. Ma, Y. Wang, Z. Zhao, B. Wen, J. He, Z. Liu, and Y. Tian, Nature 510, 250 (2014).

${ }^{3}$ L. Dubrovinsky, N. Dubrovinskaia, V. B. Prakapenka, and A. M. Abakumov, Nat. Commun. 3, 1163 (2012).

${ }^{4}$ A. Merlen, P. Toulemonde, S. Le Floch, G. Montagnac, T. Hammouda, O. Marty, and A. San Miguel, Carbon 47, 1643 (2009).

${ }^{5}$ W. Utsumi and T. Yagi, Science 252, 1542 (1991).

${ }^{6}$ L. Wang, B. B. Liu, H. Li, W. G. Yang, Y. Ding, S. V. Sinogeikin, Y. Meng, Z. X. Liu, X. C. Zeng, and W. L. Mao, Science 337, 825 (2012).

${ }^{7}$ M. Álvarez-Murga, P. Bleuet, G. Garbarino, A. Salamat, M. Mezouar, and J. L. Hodeau, Phys. Rev. Lett. 109, 025502 (2012).

${ }^{8}$ Z. W. Wang, Y. S. Zhao, K. Tait, X. Z. Liao, D. Schiferl, C. S. Zha, R. T. Downs, J. Qian, Y. T. Zhu, and T. D. Shen, Proc. Natl. Acad. Sci. U.S.A. 101, 13699 (2004).

${ }^{9}$ M. G. Yao, W. Cui, M. R. Du, J. P. Xiao, X. G. Yang, S. J. Liu, R. Liu, F. Wang, T. Cui, B. Sundqvist, and B. B. Liu, Adv. Mater. 27, 3962 (2015).

${ }^{10}$ E. D. Miller, D. C. Nesting, and J. V. Badding, Chem. Mater. 9, 18 (1997).

${ }^{11}$ W. L. Mao, H.-K. Mao, P. J. Eng, T. P. Trainor, M. Newville, C.-C. Kao, D. L. Heinz, J. Shu, Y. Meng, and R. J. Hemley, Science 302, 425 (2003).

${ }^{12}$ Y. J. Wang, J. E. Panzik, B. Kiefer, and K. K. M. Lee, Sci. Rep. 2, 520 (2012).

${ }^{13}$ X. G. Yang, M. G. Yao, X. Y. Wu, S. J. Liu, S. L. Chen, K. Yang, R. Liu, T. Cui, B. Sundqvist, and B. B. Liu, Phys. Rev. Lett. 118, 245701 (2017).

${ }^{14}$ P. J. F. Harris, Philos. Mag. 84, 3159 (2004).

${ }^{15}$ G. M. Jenkins and K. Kawamura, Nature 231, 175 (1971).

${ }^{16}$ D. F. R. Mildner and J. M. Carpenter, J. Non-Cryst. Solids 47, 391 (1982).

${ }^{17}$ P. J. F. Harris, Crit. Rev. Solid State Mater. Sci. 30, 235 (2005).

${ }^{18}$ V. V. Brazhkin, A. G. Lyapin, S. V. Popova, R. N. Voloshin, Y. V. Antonov, S. G. Lyapin, Y. A. Kluev, A. M. Naletov, and N. N. Mel'nik, Phys. Rev. B 56, 11465 (1997).

${ }^{19}$ H. Hirai, Y. Tabira, K. Kondo, T. Oikawa, and N. Ishizawa, Phys. Rev. B 52, 6162 (1995)

${ }^{20}$ M. Hu, J. L. He, Z. S. Zhao, T. A. Strobel, W. T. Hu, D. L. Yu, H. Sun, L. Y. Liu, Z. H. Li, M. D. Ma, Y. Kono, J. F. Shu, H.-K. Mao, Y. W. Fei, G. Y. Shen, Y. B. Wang, S. J. Juhl, J. Y. Huang, Z. Y. Liu, B. Xu, and Y. J. Tian, Sci. Adv. 3, e1603213 (2017).

${ }^{21}$ Y. Lin, L. Zhang, H.-K. Mao, P. Chow, Y. M. Xiao, M. Baldini, J. F. Shu, and W. L. Mao, Phys. Rev. Lett. 107, 175504 (2011).

${ }^{22}$ N. A. Solopova, N. Dubrovinskaia, and L. Dubrovinsky, Appl. Phys. Lett. 102, 121909 (2013).

${ }^{23}$ A. F. Goncharov, High Pressure Res. 8, 607 (1992).

${ }^{24}$ M. G. Yao, J. P. Xiao, X. H. Fan, R. Liu, and B. B. Liu, Appl. Phys. Lett. 104, 021916 (2014)

${ }^{25}$ N. Dubrovinskaia, L. Dubrovinsky, R. Caracas, and M. Hanfland, Appl. Phys. Lett. 97, 251903 (2010).

${ }^{26}$ A. C. Ferrari, Solid State Commun. 143, 47 (2007).

${ }^{27}$ J. Robertson, Phys. Rev. B 53, 16302-16305 (1996).

${ }^{28}$ C. Ji, V. I. Levitas, H. Y. Zhu, J. Chaudhuri, A. Marathea, and Y. Z. Ma, Proc. Natl. Acad. Sci. U.S.A. 109, 19108 (2012).

${ }^{29}$ V. I. Levitas, Phys. Rev. B 70, 184118 (2004).

${ }^{30}$ A. S. Argon and S. Yip, Philos. Mag. Lett. 86, 713 (2006). 